\title{
LE LOCAZIONI AGRARIE: DIRITTO ED ECONOMIA NELL' ANTICA ROMA
}

\section{Prof. Dr. Luigi CAPOGROSSI COLOGNESI*}

E'indubbio che la figura della locatio conductio rei si definisce concretamente e acquista rapidamente importanza negli ultimi duc secoli della Repubblica in relazione allo sfruttamento degli immobili. Del resto non può meravigliare come nella società romana, la cui base agraria tende comurque a persistere nel corso della sua storia, i frammenti dei ginristi romani raccolti nel Digesto si riferiscano a casi di locazioni di beni immobili: edifici urbari c fondi agricoli. Circoscrivendo il nostro interesse alla tarda eta repubblicana, va ancora aggiunto come, fra i giuristi di quest' epoca e quelli immediatamente successivi, della prima età imperiale, tenda ad assumere maggior rilevanza la casistica relativa alle locazioni agrarie. Va infine aggiunto - ed è questo il punto forse di maggiore interesse per noi - che proprio queste generazioni di giuristi appaiono particolarmente impegnate nella riflessione su questa figura di contratto. La percentuale dei testi riferiti ad Alfeno e a Labeone eccede di gran lunga i rapporti ordinari che incontriamo nel Digesto, quasi che, sulla locazionc degli immobili gran parte del lavoro fosse stato compiuto già con Labeone, quando in altri importanti settori ancora si era all'inizio di una strade che sarebbe giunta sino alle grandi sistemazioni di Ulpiano e di Paolo, due secoli più tardi (circa $1 / 6$ dei frammenti in sedes materiae).

Com' è noto il contratto di locazione di cose fa sorgere duc obbligazioni principali: l' obbligo, da parte del locatore, di mettere a disposizione la cosa nelle sue condizioni normali, l' obbligo al pagamento di un canone periodico predeterminato, da parte del conduttore, come corrispettivo, merces, del godimento della res. Un legato che il

* Roma "La Sapiernza" Universitesi Hukuk Fakültesi Roma Hukuku Anabilim Dail Başkan 
diritto romano ha trasmesso ai moderni diritti continentali è quindi costituito dal principio per cui l' obbligo al pagamento del canone è subordinato alla possibilità effettiva, da parte del conduttore, di fruire dell' oggetto della locazione. Se una causa di forza maggiore abbia dunque reso impossibile ciô, magari con Ia distruzione dell'oggetto stesso, allora verrà meno l' obbligo al pagamento del canone. In altro parole ciò significa che il periculum per l'oggetto della locazione resta a carico del locatore. Ancora i vecchi civilisti ci spiegavano così che "pour toute impuissance de jouir ou toute suspension de la jouissance de l' objet de la location, qui ne soit pas imputable au preveur, celui ci a clroit à la remise ou à une reduction de la merces".

Questo principio tuttora valido appare dunque chiaramentc già in testi appartenenti al primo secolo a. C e direttamente riferibili ad una delle personalità più importanti nell' elaborazione di questo aspetto del regime della locazione. Mi riferisco a Servio Sulpicio cui possiamo senz' altro attribuire ad es., D. $19.2 .27 \mathrm{pr}$. (Alf., 7 dig. $)$. Da talc testimonianza possiamo ricavare con notevolc sicurezza il criterio che, laddove lavori effettuati nell' immobile dato in locazione comportino una forte diminuzione delıa fruibilità dell' alloggio, si darà luogo alla deductio ex mercede. L' accento viene a cadere tuttavia sull' entità di questa diminuita utilizzabilità dell' oggetto della locazione, giacché, stando alla soluzioni ivi prospettata, risulta evidentc che la sospensione del pagamento del canone non è prevista per ogni inconveniente insorto nei riguardi dell' alloggio stesso.

Su questo punto, come è ovvio si tornerà in seguito più estesamente. Qui mi interessa passare a considerare il contenuto del successivo paragrafo di questo stesso brano, per noi ancor più interessante ed egualmente riferibile a Servio, in cui si considera l' ipotesi dell'abbandono della res da parte del conduttore che si sja allontanato timoris causa. Qui la perdita dell' effettivo godimento della cosa è legato a una scelta soggettiva da parte del locatario che potrà sospendere il pagamento della pensio solo nel caso in cui il timore sia giustificato da motivi oggettivi, anche se ad esso non abbia fatto seguito l'evento minacciato.

Labeone, poco più avanti, riprenderà il caso della fuga dell' inquilinus timoris causa, ma riferendosi non più all' elemento del rischio contrattuale per la temporanea o permanente perdita dell' cggetto, bensi alla respońsabjlità del conduttore per la conservazione stessa di tale oggetto. Alla fattispecie di D. 19.2.27.1 infatti si può ricondurre 
il contenuto di D. 19.2.13.7 (Ulp., 32 ad ed.,), dove è riportato un parere di Labeone in ordine alla responsabilità del conduttore derivante da danneggiamenti subiti dall'immobile dato in locazione ad opere di un esercito ostile. Se il conduttore, di fronte a questa minaccia, poteva resistere ed ha rinunciato a farlo, sarà responsabile per $\mathrm{i}$ danni che ne saranno derivati. Qui dunque la ragionevolezza del timore diventa criterio per affermare l'esonero dalla responsabilità contrattuale: in via ipotetica potremmo immaginare che Labeone aderisse all' impostazione di Servio per quanto concerne l' altro aspetto costituito dalla sospensione del pagamento del canone locativo in caso di abbandono della cosa locata per giusto timorc.

Quest' ultima ipotesi trova uno spunto sia pur lievc nel fatto che, nel passo in questione, il criterio per la determinazione della responsabilità contrattuale appare fondato sul carattere 'irresistibile' della minaccia: si resistere potuit è la formula introdotta ivi da Ulpiano a definire la soluzione del caso. Ora, appare indiscutibile l'assonanza che questo riferimento presenta con il criterio centrale che lo stesso Servio aveva affermato, in ordine alla sospensione del canone locativo, e che è ricordato in un notissimo testo di Ulpiano, D. 19.2.15.2.

Su questo passo torneremo più ampiamente nel seguito di questa analisi, quando cercheremo di cogliere dappresso l'efficacia e i limiti di questo stesso criterio della vis maior che sembra essere stato impiegato a fondare il complessivo inquadramento teorico effettuato dai giuristi tardorepubblicani in ordine alle conseguenze derivanti dal mancato o diminuito sfruttamento della cosa data in locazione. Per il momento possiamo limitarci a constatare come la enunciazione più chiara di questo principio ispiratore sia riferita a Servio in relazione alla locazione agricola. Ma esso poi sembra influenzare anche altre decisioni riconducibili allo stesso giurista: anzitutto quella ricordata in un testo di Alfeno, D. 19.2.30 pr. (3, dig. a Paulo epit.). Nel caso in cui la demolizione dell'edificio risulti necessaria, allora, si sostiene (ed anche in questo caso si tratta quasi sicuramente di un parere di Servio), sarà interrotto solo il pagamento del prezzo della locazione Se invece i lavori intrapresi dal proprietario e che impediscono il godimento dell'edificio da parte dell'inquilino, pui utili, non appaiano indispensabili, allora il dominus dovrà pagare quel di piû rappresentato non solo dalla merces, ma dall' id quod interest dell'inquilino stesso.

La particolare importanza di questo testo è data, fra l'altro, dal fatto assai significativo che il suo contenuto appare ricordato rapida- 
mente anche in un altro passo del Digesto, appartenente ad un giurista dôl II sec. d.C., Cecilio Africano, ed è esplicatimente riferito a Servin.

Il puruto clal quale il giurista per diparate una più complessa casisticà, è che l'indisporibilità dell'edificio dato in locazione comporta senz'altro la sospensione dell'obbligo del pagamento del canone d'affitto $o$, se questo c̀ già stato versato per il periodo della mancata utilizzalilitic del bei:e, la sua ripetizione da parte dell'affittuario. Se però il conduttore ha affittato l'intero edificio e, sublocando i singoli citnocula di esso a vari subaffittuari è venuto ad ottercere da tali locazioni una somma complessiva superiore a quella da lui stesso dovuta al suo locatore, at cosa avrà diritto? Sarà la mera ripetizione di quanto versato cone carore d'affitto o non sarà piuttosto anche l'id quod interest, in quesio caso commisurato alla somma totale da lui ricavata clai suoi stessi conduttori? La soluzione che lo stesso Servio con ogni probabilità ha indicato appare non univoca.

Indirettamente ma non meno significativamente riemerge anche in questo caso il criterio della vis maior della 'necessità'. Se infatti i lavori di demolizione dell', instla da parte del proprietario risulteranno indispensabili, allora egli sarà tenuto a restituire all' inquilino solo la pensio già da lui versata per il periodo in cui e venuto meno il godimento dell cedificio. Nel caso in cui i lavori siano stati intrapresi dal proprietario non pcr assoluta necessità, ma quia melius aedificare vellet, allor a l'inquilino potrà ripetere nei suoi riguardi non solo il valore dell' affitto, ma anche la cifra piu elevata corrispondentc al mancato guadagno che sarebbe derivato dal subaffitto dell' edificio. Parlavo di vis maior o di 'necessità', giacché la diversità dell' entitá della pretesa riconosciuta al conduttore dipende, nello schema di Servio-Alfeno, clirettamente dalla inevitabilità o meno del fatto dirimente alla utilizzazionc normale dell'edificio. In sostanza è la presenza di questa 'nccessità' o cli questa its maior che esclude una responsabilità per l'inadempimento dell'obbligazione da parte del locatore, dando cosi luogo allaltra situazione rappresentata dal diretto collegamento fra mercedc e fruizione della res locata, e che rientra appunto nella disciplina dei rischi contrattuali.

Questa argomentazione, che ripete e precisa quanto già indicato da Alfeno in D. 19.2.30 pr., esplicitamente riferita a Scrvio, è riportata da Fiorentino all' inizio di D. 19.2 .35 pr. Essa peraltro, lungi dal proporsi isolatamente, serve a illustrare e chiarirc un caso analogo, 
riferito ad un fondo agricolo, affrontato nell' ultima parte del precedente frammento 33, dove Fiorentino esaminava il caso di un colono cui fosse impedito l'accesso al fondo datogli in locazione. Anche qui infatti si distingueva nettamente fra il caso in cui tale impedimento fosse communque riconducibile ad una responsabilità del locatore o fosse piuttosto derivato da una vis maior ivi, ancora una volta, esplicitamente menzionata.

Et haec distinctio prosegue dunque Fiorentino all' inizio del sucessivo frammento 35 , si applica alla casistica quae a Servio introducta est, relativa alla fruibilità dell 'edificio: casistica che ho già richiamato per esteso poco più sopra. In verità a me sembra che quest' ultimo caso abbia costituito lo schema di base per la soluzione del caso considerato in precedenza: quello cioè del colono espulso dal fondo, che, con ogni probabilita, era direttamente proposto da Fiorentino. Cosi come del resto a questo stesso giurista sono riconducibili le altre fattispecie considerate nel frammento 33 , tutte riferite al problema dei rischi contrattuali, tanto nella locazione che nella compravendita. Se ben si considera dunque la struttura logica del discorso sviluppato da Africano in D. 19.2 .33 e in 35 pr., possiamo constatare come il punto di partenza sia rappresentato da una serie di casi in cui l'oggetto della locazione non è più disponibile (si fundum quem mihi locaveris publicatus sit ... si solum coruisset) cui si applica il principio ben chiaro sin da Servio che, se ciò è avvenuto senza responsabilità del locatore, sarà questione della sola restituzione della mercede, non anche del pagamento al conduttore dell'. id quod interest alla fruizione della res locativa.

Ho indugiato ulteriormente nell' analisi di questi due passi al fine di chiarire sino in fondo l'effettivo significato e l' intima coerenza della successiva considerazione che conclude questa parte della trattazione di Fiorentino, sempre in D. 19.2 .23 pr.: "quid enim interest, utrum locator insulae propter vetustatem cogatur eam reficere an locator fundi cogatur ferte iniuriam eius, quem prohibere non possit?". E' questo l' argomento con cui Fiorentino salda e assimila il caso del mancato accesso al fondo, introdotto in fine del frammento 33 , a quello dell' inutilizzabilità dell' allogio che, all' inizio del successivo frammento 35, egli riprende invece da Servio. Esplicitando maggiormente il mio pensiero, a me sembra dunque molto probabile che questa argomentazione di tipo analogico appartenga allo stesso Fiorentino piuttosto che a Servio. Non si deve infatti dimenticare come a questo giurista sia riferibile il collegamento fra $i$ due casi nel quadro di una trattazione unitaria, 
spezzata poi dai Compilatori giustinianei con l' inșerimento del brevissimo inciso gaiano costituito da D. 19.2.34.

Ma l'esplorazione, da parte di Servio, di molteplici aspetti relativi alla conservazione ed allo sfruttamento dell' oggetto locativo ha lasciato ben altre tracce nella compilazione giustitinianea. Per noi egualmente interessanti sono due brani appartenenti ad un passo che abbiamo già considerato in un' altra sua parte: D. 19.2.30.1 e 4. In cntrambi si tratta di un incendio: di un balneus nel $\S 1$, della villa rustica nel successivo $\$ 4$, Il primo caso attesta l' applicazione dei criteri che abbiamo già visto ben collaudati anche nell' ipotesi che il contratto di locazione sia stato stipulato da un magistrato cittadino a favore di tutta la comunità urbana. L' oggetto della locazione era la gratuita disponibilità di un bagro a favore di tutti i cittadini. Nel caso in cui nel corso del periodo locativo questa sia venuta meno a seguito di una vis maior - l' incendio - si potrà ripetere il canonc locativo già pagato per il periodo di mancata utilizazzione. Il "respondit" impiegato nel testo ci fa pensare, anche in questo caso, alla possibile paternità serviana della soluzione in oggetto. Più importante l' ultima parte del frammento, D. 19:2.30.4. In questo infatti noi possiamo constatare un ulteriore approfondimento della consueta tematica dei rischi contrattuali in relazione ad una specifica clausola apposta alla locatio di una villa che imponeva al colono 1' obbligo di conservazione praeter vim et vetustatem. Se l'autore dell' incendio doloso della villa sarà uno schiavo del colono, si applicherà in tal caso l'esonero di quest'ultimo dalla responsabilita per l'incendio? Rientra cioè tale atto nella sfera della wis prevista dal contratto? La soluzione negativa, egualmente attribuibile a Servio, conferma la intima coerenza del discgno cosi perseguito. La vis che rileva ai fini di questa clausola contrattuale è quel tipo di viclenza esterna che costituisce appunto l' intrinseco rischio a carico del locatore: rientra invece nella responsabilità del dominus !' atto doloso del suo schiavo - la vis da questi effettuata, dunque - proprio in considerazione del rapporto intercorrente fra $\mathrm{i}$ duc e che rende impossibile considerare lo stesso proprietario del servo come estraneo all' evento donnoso.

Non sarebbe giusto ricondurre tutta la riflessione della scuola di Servio in tema di locazione a quest' unico filone: vari brani appaiono infatti riferirsi ad altri aspetti di questa figura contrattuale, primo tra tutti un interessante testo contenuto in D.19.2.31 (Alf., 5 dig. a Paulo $e p$.), relativo all' actio oneris aversi. Colpisce tuttavia l'insistenza con cui 
l'esplorazione della casistica relativa alla persistenza dell'obligo di pagamento della mercede in relazione alla fruibilità dell'oggetto locato viene perseguita in ambito serviano. Cosi, se ci volgiamo ad un altro passo di Ulpiano, 19.2.19.1 (32 ad ed.), incontriamo nuovamente un'opinione di Servio in ordine all'esonero del pagamento della pensio nel caso in cui in un pascolo dato in locazione fossero cresciute - con l'ignoranza del locatore - erbe velenose che avessero ucciso o danneggiato il bestiame condotto a pascolare. Nuovamente l'inutilizzabilità dell'oggetto dato in locazione (in questo caso addirittura rivelatosi dannoso) rileva ai fini del pagamento del canone locativo. Anche in questo caso tuttavia entra in gioco la buona fede del locatore, giacché, ove egli fosse stato a conoscenza delle reali condizioni del campo (analogo è il caso precedente illustrato nel passo dei dolia difettosi), non si sarebbe piu nella sfera dei rischi contrattuali - o meglio, in questo caso, dei vizi occulti - sibbene in quella delle responsabilità reciproche delle parti contraenti.

Sulla rilevanza della buone fede del locatore concordano dunque con Servio anche i giuristi più tardi, da Labeone e Sabino, esplicitamente menzionati nel testo, dall' autore del passo, Ulpiano.

Non ci siamo occupati sinora di una serie di problemi che si sono posti in dottrina a proposito della sospensione del pagamento del canone locativo. Anzitutto problemi terminologici e di sostanza: abbiamo incontrato espressioni e situazioni abbastanza differenziate, ma fra loro collegate. Deductio ex mercede, remissio mercedis o reddere mercedem sono dunque espressioni che si trovano nelle fonti e che possono senz' altro presentare sfumature semantiche o riferimenti fattuali differenziati: ad es. in ordine alle due possibilità di praenumeratio o postnumeratio del canone locativo. Non sembra però utile, soprattutto dopo le precisazioni che la romanistica più recente ha saputo effettuare in ordine alle vecchie interpretazioni di Mayer-Maly e di Kaser, insistere ulteriormente su tale problematica che potrebbe; alla fine, rivelarsi sviante.

Così come solo di passaggio mi limiterò a segnalare il fatto che, alla Iuce delle nostre testimonianze, possiamo senz' altro affermare che entrambe le forme di pagamento, anticipato o successivo alla fruizione periodica del bene, erano applicate dai Romani e considerate dai ginristi sin dall' età di Servio. Stando alle pur rapide indicazioni delle fonti, si potrebbe forse immaginare che nelle locazioni d'urbane 
terdesse a prevalere la forma di pagamerto articipato, laddove in: quelle rustiche si aveva l' opposta situazionc. Nor credo comunque che i Romani e i loro giuristi si preoccupassero tanto di conformare le loro pratiche cortrattuali e l'organizzazione corcreta degli obblighi alla possibile esisterza di uno schema astratto o di modelli teorici, del resto altamente improbabili. Quello che doveva infatti rilevarc era infatti la concreta portata degli interessi e delle opporturità pratiche che cntravano ir gioco, noriché la possibile csistenza di prassi c consuctudini locali legate al contenuto ecoromico e pratico dei vari tipi di locazione.

Dalla logica di fondo che governava la disciplina delle deductiones c da molteplici spunti che è dato di cogliere nei testi dei giuristi romani si può ancora aggiungere che appare abbastanza verosimile l'idea gi accennata da vari autori, dell' esistenza di remissioni parziali del canone. Nor mi riferisco qui alla ipotesi ovvia c bene attestata nelle fonti antiche di ura deductio pro rata in relazione alla parte dell'anno di mancata utilizzazione dell' immobile, ma a quella, assai più complessa, di una remissione parziale del canone a causa della cattiva annata agricola io all' ipotesi, ancora più incerta, di una menomazione parziale nel grado di abituale friubilità dell' edificio che si contirua ad abitare). Sul punto mi sembra si abbia una relativa incestezza delle testimonianze antiche, rispetto a cui tuttavia si puó fare appello, come giustamente ha fatto De Neeve, al riferimento contenuto in un passaggio ulpianeo, D. 19.2.15.7, il cui carattere apparentemente del tutto casuale e pacifico, per ciò stesso, appare particolarmente significativo. Ma, soprattutto, nel senso di una possibile remissio pro rata anche in relazione all' entità variabile dei danni subiti dal rac: colto, potrebbe anche deporre un altro testo ulpianeo che non vedo ricordato dagli autori moderni. In esso infatti incontriamo un richiamo al possibile arbitrato boni viri in relazione al pagamento della pensio nelle cattive annate, previsto ex ante da un' apposita clausola contrattuale. E' almeno legittima l'ipotesi che tale arbitrato non riguardasse solo l'an, ma anche il quantum dell' eventuale remissio. La soluzionc positiva di tale quesito ci offre a sua volta un prezioso strumento; non solo per una comprensione più ravvicinata della natura e della logica costitutiva dello stesso istituto della remissio, - ma anche del margine di elasticità del meccanismo delle locazioni agrarie e, soprattutto, della complessita del rapporto intercorrente fra le parti contrattuali e la molteplicità dei possibili esiti. 
Sin qui dunque l' opera di Servio e della sua scuola appaiono dispiegarsi nella definizione organica e razionale di un criterio di carattere generale volto a vincolare l'obbligo del pagamento del canone locativo da parte dell' affittuario alla possibilità di una effettiva $e$ adeguata fruizione dell' oggetto della locazione stessa. Deductio e remissio mercedis, esonero dal pagamento del canone od obbligo di sua restituzione all' affittuario, sono dunque un complesso di situazioni che, in una cassistica abbastanza articolata, sembrano comunque presentare una comune matrice.

Profondamente diverse, anche se non di rado accostate nei testi giuridici romani, le ipotesi in cui la mancata o diminuita fr uizione dell'immobile derivi da un fatto arbitrario o ingiustificato di uno dei due contraenti. Questa generale ipotesi che attiene non piu alla problematica del periculum contrattuale, ma al campo della responsabilità, ricomprende a sua volta due situazioni opposte. Da una parte infatti rileva un eventuale danno derivante all' oggetto locativo, ove ciò possa in qualche modo essere imputabile alla condotta del conduttore; dall' altra è invece da considerarsi l' ipotesi di un danno derivante al conduttore dalla diminuita o mancata fruibilità dell' oggetto in questione causato da un atto arbitrario imputabile al locatore stesso. Il discrimine tra queste due diverse fattispecie - il periculum e la responsabilità - è ben chiaro sin dai veteres èd appare chiaramente tracciato nei testi che siamo venuti esaminando.

Esso dunque è costituito, come abbiamo visto, dalla evitabilità o meno dell' evento dannoso. L' elemento della responsabilità delle parti contrattuali - e la conseguente cessazione della disciplina del rischio contrattuale - ̀̀ dato appunto da un fattore soggettivo che interviene. Chi voglia ripristinare un edificio senza che ciò risulti indispensabile per la sua immediata conservazione, chi abbia lasciato pascolare le pecore del conduttore sul proprio fondo dove crescevano herba mala e di ciô, sapendolo, non lo avesse avvertito, chi abbia infine abbandonato l' edificio preso in locazione alla devastazione di un esercito ostile, senza dar notizia al proprietario del pericolo incombente, tutti costoro hanno contribuito a ingenerare un evento dannoso di volta in volta a carico del conduttore o del locatore e sono quindi responsabili in base agli obblighi contrattuali.

Vi è tuttavia un momento in cui le due figure si intrecciano, sia pure in maniera del tutto estrinseca. Mi rifersico al fatto che la vis maior è richamata tanto nella disciplina della responsabilità che in 
quella del periculum contrattuale. Nel primo caso infatti essa serve a sancire la imputabilità o meno del fatto dannoso al comportamento dell' uno o dell' altro contraente, e serve quindi a definire il territorio stesso della responsabilità contrattuale rispetto a quello relativo al regime dei rischi. Ma la vis maior appare poi richiamata dagli stessi veteres a regolare la imputazione dei rischi all' una o all' altra parte del contratto, operando cosi anche, in modo esclusivo, all' interno del territorio del periculum. Delle specifiche testimonianze relative alla disciplina di quest' ultimo aspetto si tratterà immediatamente di seguito, vorrei tuttavia affermare sin da ora che l'impiego di questo strumento concettuale constituito dalla vis maior risulta più efficace ai fini della separazione dei rischi dalla responsabilità ex contractu piuttosto che per distinguere i casi di riferimento dei rischi stessi a carico del conduttore o del locatore.

Dal punto di vista economico sociale appare d'altra parte evidente che 1' utilitas inerente al contratto in questione è costituita dallo sfruttamento del bene secondo le sue specifiche caratteristiche: se si tratta di un edificio urbano, essa consisterà nel fornire al conduttore l'abitazione, il fondo rustico sarà invece oggetto di uno sfruttamento agricolo o pastorale. Per l'immobile urbano è abbastanza netta l'identificazione del mancato godimento del bene con la sua inutilizzabilita' dal punto di vista abitativo. Ma più complessa è la situazione dei fondi rustici. Qui infatti non gioca solo il caso di una loro inutilizzabilità assoluta ('invasione di un esercito, la distruzione del fondo), ma anche una non rispondenza di tale bene agli usi e ai rendimenti abituali previsti in proposito. Se nel prato crescono erbe velenose, se le culture di quell'anno sono devastate in modo irrimediabile da eventi esterni, cui resisti non potest, egualmente le possibilità di sfruttamento del bene, 0 meglio le conseguenze economicamente vantaggiose da ciò derivanti, sono sospese per un certo periodo di tempo $\mathrm{Il}$ periculum, in questo caso, ricade sul locatore, sul dominus del bene stesso.

E qui torniamo dunque al brano ulpianeo che abbiamo già sfiorato, in cui appare riferito per esteso, ancora una volta, il pensiero di Servio in ordine alla sospensione del pagamento del canone a seguito di eventi eccezionali che abbiano turbato le normali attività agricole. Mi riferisco a D. 19.2.15.2, che converrà riportare per esteso:

$S i$ vis tempestatis calamitosae contigerit, an locator conductori aliquid praestare debeat; videamus. Servius omnem vim cui resisti non potest, dominum colono praestare debere ait ut puta 
fluminum graculorum sturnortm et si quid simile acciderit, aut si incursuus hostium fiat: si quid tamen vitia ex ipsa re oriantur, haec damno coloni esie veluti si vinum coacuerit, si raucis aut herbis segetes corruptae sint. Sed et si labes facta sit omnemque fructom tulerit, damnum coloni non esse, ne supra damnum seminis amissi mercedes agro praestati cogatur. Sed et si uredo fructum oleae corruperit aut solis fervore non adsueto id acciderit, damnum domini futurum: si vero nihil extra consuetudinem acciderit, damnum coloni esse. Idemque dicendum, si exercitus praeteriens per lasciviam aliquid abstulit. Sed et si ager terrae motu ita corruerit, ut nusquam sii, damno domini esse: oportere enim agrum praestari conductori, ut frui possit.

Certo, il testo non è esemplare e si puo senz' altro registrare la presenza di irregolarità formali e di cadute stilistiche che possono far pensare a tagli o ad altri possibili interventi postclassici. Insistere troppo su questo aspetto significa perô perdere di vista I' interna çoerenza del discorso ivi riportato e la sua corrispondenza con quanto è stato dato sinora di cogliere in ordine alle conseguenze della mancata utilizzazione della res. E' vero che rispetto alla problematica che abbiamo rapidamente esaminato nel corso del precedente paragrafo, qui si ha a che fare con il presupposto diverso rappresentato dall' assenza non già della res ma dei risultati utili ricavati dalla sua utilizzazione. E' però anche vero che identico appare il mcdo di procedere del giurista repubblicano, volto appunto a individuare anche in questo caso un elemento significativo di rischio, tale da essere ricondotto al principio del periculum locatoris. Ma non mi fermerò ancora una volta su una testimonianza tanto importante quanto nota e battuta dalla nostra tradizione di studi cui, in linea di massima, converrà rinviare il lettore.

Qui mi interessa piuttosto ribadire alcuni nodi problematici solo apparentemente risolti dalla cartesiania semplicità della contrapposizioné vis cui resisti non potest - vitia ex ipsa re e che già erano stati individuati con chiarezza negli studi di questi anni. All' uopo sgombriamo immediatamente il campo da alcune distorsioni, in primo luogo e piu grave di tutte l' idea sostenuta da alcuni recente autori, secondo cui tale riferimento all' ipsa res evocherebbe quei vizi verificatisi nell'attività agricola a seguito di un comportamento inadeguato o colposo 
del conduttore e che quindi, con una condotta più atlenta, avrebbero potuto essere evitati. E' questa un' idea che, a ben considerare, reintrodurrebbe nel frammento in questione il problema della responsabilità accanto a quello dei rischi contrattuali, spostando cosi una prospettiva che, a mio giudizio appare invece esclusivamente incentrata sulla questione dei limiti in cui $i$ rischi relativi al pacifico sfruttamento della res locativa siano a carico del locatore. In verità l'impostazione che emerge dal frammento e che è senz' altro da attribuire a Servio mi sembra abbastanza chiara: di fronte all' evento straordinario e dannoso, cui resisti non potest non vi è il danno provocato da un comportamento inadeguato dell' affittuario, da una sua insufficienza nella coltivazione del campo, bensi quel variare dei risultati e quegli insuccessi che sono intrinseci alla natura stessa dell' attività in questione: che, appunto, derivano ex ipsa re, per il carattere sostanzialmente aleatorio e variabile dell' agricoltura con i suoi alterni risultati. Concludendo dunque mi sembra si possa sostenere che il valore cosi assunto in questo passo dal riferimento all' ipsa res si ponga in termini alternativi al carattere straordinario degli eventi che sono ricondotti all' interno dell' opposta categoria della vis maior, costituendo quindi i poli opposti di un sistema.

Il problema che Servio cerca di dipanare con la casistica che illustra le due ipotesi alternative e alla quale forse anche Pomponio o Ulpiano possono avere aggiunti altri esempi, è appunto la determinazione del discrimine fra due tipi di esiti svantaggiosi. Quelli che rientrano in una gamma di variazioni che possono essere considerate 'normali' c quelle che assumono un carattere eccezionale. Nella problematica che il nostro testo affronta in quella forma abbastanza disordinata che ha giustificato i sospetti, peraltro eccessivi, che sono stati avanzati su di esso, troviamo comunque un filo logico di cui darò rapidamente conto, tenendo conto, del resto al vasto lavoro esegetico già da altri effettuato in proposito. Tale casistica dunque appare svilupparsi proprio in funzione di una più chiara evidenza di quella polarità cui si è fatto cenno. Cosi da una par te troviamo indicati gli eventi eccezionalmente gravi ecertamente non imputabili ad una cattiva coltivazione da parte del colono: la labes intervenuta a impedire ogni raccolto, una gravissima siccità contrapposta appunto alla consuetudo delle annate buone che si seguono alle cattive, cosî infine l' imperversare di un esercito ostile, anche qui contrapposto al danno episodico arrecato dal furto di un qualche soldato isolato, sia esso amico o nemico, ma egualmente lascivus. 
Naturalmente la contrapposizione fra modifiche tollerabili. e fatti imprevedibili e troppo rilevanti rispetto al contenuto stesso della locazione non riguarda solo lo sfruttamento agricolo. Questa stessa polarizzazione infatti l' abbiamo egualmente incontrata in relazione alla casistica relativa alla persistenza dell' oggetto della locazione che abbiamo considerato nel corso del precedente paragrafo. Dove finisce infatti il piccolo incommodum che non dà luogo alla deductio ex mercede, nell' uso dell' abitazione e dove ha inizio quella degradazione dell'allogio che giustifica invece tale deductio? E' questo un punto che abbiamo visto affidato ad una valutazione di buon senso - la scomodità e la riduzione di abitabilità tollerabile rispetto alla sostanziale anche se non totale inagibilita dell' alloggio - ma per ciò stesso lungi dall'essere risolutrice di ogni dubbio ed incertezza. Del resto non si potrebbe in qualche misura applicare la stessa nozione dell' ipsa res anche alla ordinaria variazione di comodità e fruibilità degli alloggi (delle rotture o deterioramenti di parti accessorie dell' edificio etc....) e contrapporre poi, anche per le locazioni urbane, questa a quella vis maior che, del resto, abbiamo già visto esplicitamente applicata a tali fattispecie?

Egualmente, ne caso di remissio affrontato in D. 19.2.15.2, la linea di confine fra le due opposte ipotesi, l'ex ipsa re e la vis cui resisti non potest, non è chiarita in modo sicuro né da Servio, nel corso della pur ampia casistica che viene dipanando, e neppure da Pomponio e Ulpiano che, in fondo, sulla logica da lui seguita sembrano appiattirsi. Ed è per questo che, nel corso delle generazioni successive, la discussione non verrà a svilupparsi intorno alla logica che ispira la generale architettura già definita da Servio, ma proprio in ordine a quella zona grigia e indefinita che si colloca fra i due pali opposti della catastrofe o dell' ordinaria amministrazione.

Se partiamo da tali premesse, allora la sequenza in cui si collocano $\mathrm{i}$ testi canonici relativi alla remissio mercedis - D. 19.2.15.2, e poi D. 19.2.25.6, seguito da D. 19.2.15.3, 4 e 5 - assume nuova evidenza. $\mathbf{E}$ assai maggiore pregnanza e coerenza assumono altresî quegli interventi imperiali in materia che non mi sembrano per nulla avere quel ruolo affatto innovativo in materia, con la introduzione ex novo della stessa remissio mercedis, che, come s' e già accennato, è stato suggerito da alcuni romanisti.

E, cosi pienamente comprensibile il motivo per cui, circa di due secoli dopo Servio, tanto Gaio che Papiniano continuano a interrogarsi 
sui casi in cui potrà ammettersi in concreto la remissio mercedis. L' importanza maggiore di D. 19.2.25.6 (Gai, 10 ad ed. prov.), esula dal nostro orizzonte immediato, consistendo sicuramente nella menzione della colonia partiaria: 1' unico riferimento chiaro che a tale istituto si incontra nei testi giuridici, singolarmente reticenti in proposito. Anche in ordine ai nostri problemi vi è però un' indicazione importante in questo passo che va sottolineata. Si tratta del fatto che Gaio tende a spostare, o comunque ad arricchire la prospettiva battuta a suo tempo da Servio e seguita verosimilmente da Pomponio e dallo stesso Ulpiano. $\mathrm{E}$, infatti il significato della vis maior che ora viene a qualificarsi anche in relazione alla dimensione quantitativa del fenomeno: plus quam tolerabile. Ma la dilatazione della vis maior avvienc anche in un altro senso, ricomprendendo così non solo gli eventi che verranno addebitati al locatore (perchè 'intollerabili'), ma anche quelli, 'tollerabili' che resteranno quindi a carico del conduttore. In tal senso la vis maior non è più contrapponibile all' ipsa res, riguardando ogni fatto non dipendente dalla condotta delle parti. E' questa certo una innovazione che corregge la relativa indeterminatezza concettuale che abbiamo potuto cogliere alla base della contrapposizione vis maior - ipsa res di Servio ed appare quindi almeno legittima, sc non necessaria sotto il profilo logico. E tuttavia non può sfuggire il fatto che, in tal modo, estendendosi il riferimento della vis maior anche alla gamma ordinaria delle fluttuazioni agrarie, si è finito col perdere in termini di efficacia operativa ciò che si era acquisito come coerenza e chiarezza concettuale.

Ad avvicinare poi lo schema della locazione a quello della colonia parziaria vi è ancora un altro elemento che vorrei rapidamente sottolineare. Mi riferisco alla giustificazione addotta da Gaio a spiegare l' attribuzione dei modici danni al conduttore. Che $\dot{e}$, di nuovo il carattere aleatorio dell' attività agricola, il quale permette anche l' immodicum guadagno che resta pur esso a vantaggio totale del conduttore. Ed è questo margine maggiore di alea (anche in senso positivo dunque) che caratterizza l' un tipo di contratto rispetto all' altro, la colonia parziaria appunto, dove sia guadagni che perditc appaiono ripartite in proporzione fra le due parti, quasi societatis iure. Verrebbe fatto di pensare che 1 ' automatismo della ripartizione dei rischi proprio della colonia parziaria in qualche modo si riflettesse sulla tendenza a dilatare il sistema delle remissiones nella locazione facendo cosî emergere, anche in quest' ultima figura, un elemento di proporzionalità 
delle perdite rispetto alla ripartizione della rendità fra le due parti in gioco. E' comunque abbastanza chiaro che, man mano che si espande l' ambito di applicazione delle remissiones, viene a diminuire la distanza fra i due tipi di contratti, accentuandosi, sia pure parzialmente anche per la locazione il rapporto fra rese complessive del fondo e livello della remunerazione del dominus.

Relativo appare il vantaggio offerto dallo schema gaiano: la tollerabilità o meno dell' évento dannoso infatti comporta un margine di indeterminatezza e di opinabilità non minore del richiamo all'ipsa res serviana e non può considerarsi uno strumento di mediazione particolarmente efficace nella costante tensione che veda contrapposti gli interessi degli affittuari a quelli dei proprietari fondiari. Perchè appunto questi vengono a confliggere in relazione a quelle situazioni di incertezza, a quelle aree 'grigie' che ho richiamato, in cui lo stabilire se la crisi ha carattere eccezionale o meno assume il carattere di una scelta con un margine di arbitrarietà, contestabile quindi e contestata effettivamente da chi ne ha 1" interesse.

Di qui dunque l' esigenza di mediazioni ed arbitrati; sino appunto alla contesa giudiziaria, ma anche il ruolo di supremo arbitro di questi conflitti che verrà ad assumere il Principe; nọn già autore di un. nuovo strumento di 'politica economi a e sociale' o di un nuovo istitùto giuridico, ma garante del funzionamento dei sistemi antichi in ragione degli ampi margini di incertezza che essi da sempre comportavano.

Su alcuni testi relativi a rescritti imperiali mi riservo di tornare più avanti, per ora mi concentrerò sú D. 19.2.15.3 (Ulp., 32 ad ed), in considerazione di uno specifico elemento in esso contenuto. Qui infatti l' Imperatore è chiamato a dirimere una controversia che riguarda l' applicabilità o meno della remissio al caso di una perdita del raccolto per un incendio sviluppatosi ne fondo. La soluzione adottata è che si praedium coluisti, propter casum incendii repentini non immerito subveniendum est. Diversamente dai tentativi di spiegazione ancora di recente proposti dai commentatori, a me sembra che la difficoltà della scelta sottoposta al Principe e la logica che ha guidato quest' ultimo siano da collegarsi al problema di fondo della ambiguità dell' incendio. Certo, 'causa di forza maggiore', ma non cansa ingenua e atta sicuramente ad esonerare la responsabilità umana, al contrario. $\mathrm{Ri}$ cordiamo del resto D. 19.2.30.4, dove appunto di un incendio si trat- 
tava, ma appiccato dolosamente dallo schiavo dell' affittuario, e talc quindi da coinvolgere direttamente la responsabilità di quest'ultimo.

Un incendio sviluppatosi in un terreno agricolo ingenera immediatamente - oggi come duemila anni or soro - il sospetto di un'incuria del contadino: si pensi solo alla antica pratica della bruciatura delle stoppie, atta a rigenerare la fecondità della terra, ma anche a dilatare incendi a seconda del vento e della condizione generale di siccità dei campi. E si pensi quanto difficile sia accertare, comunque, eventuali responsabilità in proposito: l' unica sarà dunque rifarsi alla qualità generalc e collaudata dell' agricoltore. Si praedium colusti ...; se i tuoi precedenti insomma depongono a tuo favore scatterà una presunzione di irresponsabilità rispetto all' incendio e solo allora questo evento, sottratto alla sfera della responsabilità, rientrerà nella problematica dei rischi contrattuali, qualificandosi così come un caso di vis maior, tale da legittimare la remissio. Tanto più che, in caso di precedente incuria da parte del conduttore, l' incendio del campo potrebbe fare insorgere il sospetto di un atto doloso volto a sottrarsi agli obblighi contrattuali il cui adempimento poteva essere reso difficile o impossibile proprio dalla cattiva coltivazione.

Comunque sia, questo intervento imperiale si colloca chiaramente all' interno di una problematica tipica del nostro istituto e che investe la questione centrale se una data fattispecie rientri o rell' ambito di applicazione della remissio, o meglio, se essa ricada rell' ambito della responsabilità o in quello del periculum. Secondo la mia ipotesi il motivo di dubbio consisterebbe nella possibile presenza di un comportamento irregolare del colono che escluderebbe a priori la stessa nozione di zis maior. In questo caso dunque l' incertezza non attiene alla dimensione quantitativa del danno subito dal raccolto, ma alla causa ultima di questo: di nuovo si passerebbe dal problema dell' attribuzione dei rischi a quello della definizione della responsabilità.

Riferito invece alla questione che resta centrale della entità del danno appare il paragrafo immediatamente successivo a quello ora esaminato, nel quale Ulpiano cita un interessante orientamento di Papiniano. Senza entrare direttamente in merito all' ertità della crisi propter sterilitatem, per cui è concessa la remissio mercedis questo giurista sostiene che di questa stessa remissio dovrà tenersi conto negli anni successivi. Ove infatti in questi le rese siano elevate, l' affittuario sarà tenuto nei riguardi del locatorc anche per la pensio a suo tempo 
non pagata. Ed infatti, conclude Papiniano, la remissio non ha carattcre di donazioné, ma quasi di transactio.

Torneremo in seguito su quest' ultimo punto che ha un valore strategico per una più piena ccmprensione dei complessi meccarismi, anche di carattere meramente sociale, che entrano in gioco con questo istituto. Qui mi interessa rilevare anzitutto un sotterraneo collegamento che potrebbe sussistere fra il criterio fatto valere da Papiniano e il testo gaiano ricordato poco più sopra. In questo infatti il riferimento ai possibili grandi guadagni, superiori alla media, da parte del conduttore a giustificazione dei piccoli rischi a suo carico, apre la strada ad una valutazione piu ampia dell' everto singolo costituito dalla crisi di un raccolto. Si definisce infatti un potenziale rapporto fra l' andamento di una stagione e quello di altre all' interno della stessa vicenda contrattuale, laddove appurto la diminuita resa di una data annata viene associata agli evertuali grardi guadagni di altre stagioni. Sotto questo profilo I' impostazione di Papiniano appare del tutto omogenea a quella che è stato dato di cogliere nel precederte testo di Gaio e ne costituisce solo un' applicazione pratica.

Ciò detto, va anche sottolhheata adeguatamente la difficoltà intrinseca alla scluzione cosi proposta: come si valuterà il tipo di resa che possa giustificare il recupero della precedente remissio, a quali medie, se del caso, fare riferimento? I problemi d' ordine strettamente quantitativo che derivavano dalla difficoltà di classificare quella zona intermedia fra l' ordinaria resa del fordo e la catastrofe eccezionale ed alla quale ho fatto a più riprese riferimertc, si ripropor.goro, in modo se possibile ancora più evidente, anche nel caso che, ai fini della remissio entri in gioco un più ampio arco di tempo per valutare la resa complessiva del fondo. Proprio per questo del resto, mi sembra che, se questo dilatato criterio di valutazione da una parte appare spostare il rapporto fra locatore e conduttore a favore del primo, rischia poi di accentuare invece che sciogliere il potenziale livello di conflittualità fra le due parti.

A questa conflittualità è giunto il momento di tornare per penetrare più a fondo nel complesso gioco interattivo fra istituzioni legali e meccanismi sociali. Volendo partire da una banalità possiamo dire che proprio la casistica sinora esaminata evoca essa stessa la difficile strada' percorsa dai Romani, probabilmente prima in occasione di pratiche consulenze e di discussioni giudiziarie che non in sede di riflessione teoretica, per determinare l' ambito di applicazione della 
remissio. Ma di queste difficoltà e del concreto scontro di interessi che queste accentuavano e da cui, insieme, ne venivano inaspriti, a me sembra sia più immediata testimonianza quel gruppo di testi in cui sono riportati i diversi interventi imperiali. Lungi dall'introdurre movi istituti (che sigrifica del resto fondare questa ipotesi sulla sola innovazionc processuale, quasi che il tramonto del processo formulare non fosse fenomeno affatto generalizzato per tutto l' ordinamento giuridico romano?) testi come C. 4.65.8, e 19, e D. 19. 2.15. 5, confermano la continuità nel tempo di una insistita esplorazione dei confini intrinseci alla remissio mercedis, stimolata appunto anche dalla ricorrente pressione esercitata dagli affittuari per dilatare l'applicazione delle remissiones. Ciò è del tutto evidente in D. 19.2.15.5, laddove si menziona il rigetto, da parte del Principe di due richieste che chiaramente appaiono al di fuori dei criteri elaborati dalla giurisprudenza repubblicana ed imperiale in tema di remissio.

Non basta un cattivo raccolto - per definizione rientrante in quelle fluttuazioni tipiche dell" "ipsa res" serviana - per legittimare alla remissio: ma ancor meno si giustificherebbe questa nel caso di un raccolto evidentemente insoddisfacente per la vecchiaia delle vigne. Al momento del contratto la condizione dei vigneti era evidente alle parti e poteva quindi essere assunta come parametro per la definizione dell' ammontare della merces. Non interviene dunque alcun nuovo evento, alcun fattore esterno alle vicende contrattuali che ecceda $i$ normali rischi agrari. Anzi, a ben vedere, in questo caso non si può neppure parlare di un 'rischio' derivando la povertà dei frutti dalla condizionc obiettiva delle vigne, del tutto accertabile $e x$ ante. Ha dunque senz' altro ragione De Neeve nell' escludere, contro precedenti interpretazioni, che siffatte testimonianze possano farci supporre un orientamento imperiale più favorevole agli affittuari: al contrario. almeno questo testo, come già la precedente testimonianza di D. 19.2.15.3, sembra confermare un orientamento degli imperatori strettamente coerente con l' impostazione che già era stata data in eta repubblicana e che $\mathrm{i}$ giuristi imperiali si limitavano a precisare e ad approfondire.

Più complesso il discorso relativo alle più tardive costituzioni di Alessandro Severo e di Diocleziano, in un periodo in cui ormai lo tradizione classica era al tramonto. Questo non certo, sia ben chiaro, perché i due imperatori sembrino in qualche modo muoversi al di fuori degli schemi già definiti dai classici per la remissio mercedis, 
ma perchè, più che di un accentuarsi del conflitto fra le parti contraenti sembra qui evocarsi con maggiore evidenza quel contesto di pratiche agrarie e di concreti assetti di interessi che il più elevatc livello di astrazione dei testi dei giuristi classici in qualche modo tende ad attemare.

Il primo e più importahte aspetto che mi interessa mettere in adeguata evidenza è la presenza, nei due rescritti citati, di un elemento sinora sfuggito al nostro orizzonte: I mores regiones. Nell' un caso noi vediamo che queste consuctudini locali possono giungere sino a modificare i principi che regolano d'ordinario il contratto, spostando a carico dell' affittuario il rischio per la perdita del raccolto. In tal caso queste pratiche avranno valore legale non meno di apposite clausole contrattuali. Importante ancora questo stesso rescritto di Alessandro Severo giacchè vediamo in esso recepito quel principio enunciato più di mezzo secolo prima da Papiniano, secondo cui la remissio ha luogo solo ove la sterilitas di un anno non possa essere compensata dalla ubertas degli altri anni della locazione.

Del tutto coerente con la logica generale del contratto la conclusione di Diacleziano in ordine a particolari casi di remissiones effettuate contra legem contractus atque regionis consuetudinem. In tal caso il comportamento del locatore assume il carattere effettivo di liberalità e non costituisce quindi un precedente che possa essere fatto valere nella disciplina di analoghi rapporti contrattuali. Anche qui insorge il sospetto che, diversamente, a questo precedente si siano appellati i piccoli affittuari interessati a consolidare questo margine di vantaggio, estendendo a tutti la decisione troppo benevola di qualche proprietario locale. Più incerto resto invece di fronte alla prima parte del testo, laddove infatti sembrerebbe enunciarsi un principio di grandissime conseguenze e cioè che l' autonomia delle parti potrebbe trovarè un limite nelle consuetudini regionali. Tale infatti parrebbe il senso dato alla generale enunciazione d' apertura secondo cui la fides contractus non sarebbe salvaguardata ove il suo contenuto andasse contra consuetudinem regionis. Ma verosimilmente l' espressione imperiale va piuttosto riferita ad un problema di interpretazione del contratto che non a un preciso vincolo imposto all' autonomia della volontà contrattuale.

Invero la menzione di queste consuctudini regionali in qualche modo appare troppo episodica e limitata perchè si possa da ciò tentare una più complessa c generale ricostruzione dei caratteri dell'- 
intera parabola di tale istituto. Il fatto tuttavia che tale richiamo sia effettuato in maniera così pacifica, non può non far sospettare che si trattasse di tradizioni diffuse e ben radicate nelle varie parti dell'Impero. E questo mi induce ad avanzare alcune ipotesi circa il modo in cui il sistema delle remissiones ha preso sostanza ed a trovato successivamerte una sua prolungata applicazione all' interno delle affittanze agraric.

Volgendoci dunque verso un' età assai più antica, io tenderei ad immaginare che l' irtroduzione della remissio mercedis ad opera dei giuristi tardo repubblicani in collegamento alla più vasta problematica dei rischi contrattuali possa essere stata influenzata dalla presenza di pratiche forse arcora più antiche, di comportamenti concreti delle parti e di clausole contrattuali o di successive pattuizioni e 'transazioni' fra gli interessati. Su questa base si sarebbe poi sviluppata la consapevole elaborazione dei giuristi, orientati così a dilatare la stessa nozione di periculum locatoris con l' assimilazione della mancata produzione agraria al deterioramento o alla scomparsa dell' oggetto della locazionc.

D' altra parte vi è certo una storia della locazione, anteriormente a Servio, che sfugge al nostro sguardo. Gli stessi fattori genetici di tale istituto sono incerti, arche se tuttora sarei propenso a pensare che il collegamento proposto a suo tempo da Mommsen fra le locazioni pubbliche e la genesi della locatio conductio rei sia stato negato dai moderni con troppa facilità e, communque, a favore di ipotesi ancora meno probabili,

Il fatto che Servio si impegni cosi a fordo sia in relazione al problema specifico del deperimento dell' oggetto della locazione che per quello della mancata produttività di tale oggetto a seguito di eventi eccezionali potrebbe indurre ad avanzare alcune ipotesi. Anzitutto che già prima di questo giurista si fosse venuta delineando una problematica che abbracciava sia I' un tipo di casi che l' altro. Noi non disponiamo, su questi aspetti, di testimonianze relative a Quinto $\mathrm{Mu}$ cio o alle generazioni ancora più antiche di giuristi: è però possibile che già allora le crisi agricole di carattere eccezionale fossero state assunte all' interno della dicsiplina dei rischi contrattuali. $\mathrm{Va}$ anche sottolineato, d' altra parte, come remissioni del pagamento dei canoni locativi urbani appaiano introdotte autoritativamentc, sin dalla fine della repubblica, con appositi provvedimenti normativi. Ma sopra- 
tutto è importante ricordare come Cicerone, nel $D e$ officiis menzioni la remissio a favore dei propri coloni come pratica affatto nota e consolidata. Anche il parallelo richiamo alle possibili remissioni effettuate a favore dei grandi appaltatori pubblici, legati allo Stato con un vincolo analogo al privatistico contratto di locazione è estremamente significativo, giacchè ci introduce in un più ampio panorama in cui elementi di valutazione latamente politica appaiono giocare un ruolo non solo in quest' ultimo settore, ma anche nei rapporti privatistici (mi riferisco alle locazioni urbane), permettendo cosi di inquadrare la stessa prospettiva serviana in un quadro più articolato.

Queste considerazioni mi inducono dunque a sostenere che, con molta probabilità, già prima di Cicerone e di Servio 1' applicazione del contratto di locazione ai rapporti agrari, attraverso l' affitto di fondi o di parti di fondo doveva essere diffusa. Ma soprattutto appare abbastanza verosimile che, già in tale epoca affittuari e proprietari dovevano conoscere ed applicare la pratica di una sospensione o di una diminuzione del canone pattuito nel caso di gravi crisi agricole. Ed è questa idea della 'crisi'; piuttosto che il mero riferimento alla mancata utilizzabilità della res che potrebbe avere costituito il riferimento concettuale (se mai se ne sia effettivamente avvertita la necessiatà) per quegli interventi politici di emergenza, ricordati dagli antichi, volti a sospendere o ad alleviare il pagamento degli affitti urbani.

Gli accordi e le pattuizioni private che dovevano conformarsi a pratiche diffuse nei vari distretti agrari e che, a loro volta, dovevano rafforzare ed ampliare l'efficacia di queste ultime non potevano ovviamente restare estranee alla visuale dei giuristi nel momento stesso in cui questi si vennero impegnando più in generale sulla figura della locatio conductio rei. Già nel corso della prima generazione dei veteres, della cuì riflessione in proposito il ricordo è pervenuto sino a noi, vediamo cosi l' istituto delle remissiones agrarie già bene al centro dell'attenzione. Ed in tale contesto gli strumenti interpretativi a disposizione rendevano possibile l' inquadramento di tale pratica all' interno del più vasto regime del periculum locatoris con quell' assimilazione della mancata resa del fondo alla inutilizzabilità della cosa di cui si è già discorso. Questa operazione che, logicamente potrebbe apparire un posterius rispetto alla disciplina del caso centrale della distruzione o dell'indisponibilità della cosa in ordine al sistema deł periculum, appare invece contemporaneà, se non addirittura anteriore proprio per l'importanza di comportamenti pratici e di concrete modifiche contrat- 
tuali che con ogni probabilità già si erano diffusc nell' ambito dei rapporti agrari romani fra il secondo e il primo secolo a.C.

Anche alla luce di questa ipotesi assume maggiore evidenza l'insieme di riferimenti che ho effettuato, nel corso di queste pagine, al valore legale e cogente per le parti dell' istituto della remissio mercedis. Ho già accernato al fatto che, da to lo squilibrio sociale esistente assai spesso fra proprietari fondiari e affittuari, il carattere di mera transactio vincolante per le parti fossc parzialmente celato sotto l' apparenza. della concessione unilaterale, di una benevola decisione a seguito delle presssanti richieste dei ccntadini in difficoltà. Questo, fra l' altro, come abbiamo visto, giustifica proprio la reazione dei giuristi e del Principe c trova alcune chiare conferme in altri tipi di testimonianze.

La prima mi sembra possa essere costituita da un ben noto passo di Columella, giustamente utilizzato da alcuni romanisti nclla loro interpretazione del nostro istituto. In questo testo incortriamo dunque I' esplicito consiglio rivolto al proprietal io di non essere esigente nel pretendere il regolare pagamento del canone dai suci affittuari, mostrandosi piuttosto esigente per quanto concerne la buona coltivazione del fondo. Questa mancata pressione finanziaria sul colono necessariamente è destinata ad assumere la forma, sotto il profilo strettamente lcgale, dei reliqua o delle remissiones sia pure parziali dei canone locativo. Ora la situazione così rapidamente evocata da Columella parrebbe avvicinarsi a quanto abbiamo incontrato in C., 4.65.19, laddove, per l' appunto, sono ricordate delle remissioni effettuate come puro atto di liberalità del locatore e non anche vincolate alla $l \in x$ contractus o alla regionis consuetudo. Si tratta, anche in questo caso, di una gamma di situazioni e di casi particolari difficilmente imprigionabile all' interno di schemi formali troppo rigidi. Dove finisce la benevolenza, la 'ricattabilità' del dominus rispetto alle qucrimonie e alla effettiva miscria del suo affittuario e dove ha inizio un suo vero e proprio obbligo deducibile in giudizio? La risposta, lo abbiamo già visto, non è facile perchè e collcgata direttamente al difficile problema cha abbiamo esaminato nel corso dei due precedenti paragrafi del tipo di danni ai raccolti che legittimano alla remissio in questione. Proprio in ragione di quella "zona grigia" intermedia di cui ho parlato a suo tempo, prende corpo la realtà dei comportamenti concreti dei proprietari. Certo, regolati e disciplinati in parte dalle pratiche locali, dalle "consuetudini regionali". di cui ci parlano le fonti antiche, ma che inevitabilmente sono anche espressione di scelte individuali, 
di una episodicità che non può non esaltare, in ultima analisi, il forte elemento soggettivistico che cosî si esprime, assumendo quindi sovente il carattere paternalistico di una benevolenza più o meno forzata. Una benevolenza e concessioni antiche forse quanto le stesse affittanze agrarie e che, almeno tendevano a compensare sul piano della lealtà di comportamenti e della subordinazione quei-vantaggi economici cui temporaneamente si rinunciava.

Quella benevolenza, a ben. vedere, che si esprime, nella forma un po' annoiata, addirittura lievemente esasperata, dell' epistolario pliniano. I brani delle lettere di Plinio il giovane cui faccio riferimento sono ben noti e, oserei dire, persino troppo sfruttati. Con questa mia ultima affermazione in forma paradossale mi interessa sottolineare che è difficile in ultima analisi valutare il carattere esemplare di questa testimonianza non dico per un intero periodo della vasta parabola dell' agricoltura romana, ma anche per l' insieme di situazioni regionali che costituiscono la realtà agraria dell' Italia imperiale. Communque sia, queste lettere attestano non solo le ripetute remissiones che Plinio si vede "costretto" a concedere dalle continuae sterilitates, ma anche qualcosa di più.

Esse infatti ci introducono in un sistema organizzativo che, malgrado le interne difficoltà che forse negli anni di redazione dell' epistolario si sono venute accentuando, presenta alcuni punti di forza che ne garantiscono la durata. Il primo è dunque costituito proprio dalla relativa elasticità del sistema delle affittanze - mobilità dei coloni, loro trasformazione in mezzadri etc. - il secondo mi sembra rappresentato dalla presenza di un aspetto organizzativo nella gestione delle affittanze fondato su un meccanismo più o meno esplicitamente gerarchico, dove il grande proprietario tende ad assumere un potere di controllo e dí intervento che si fonda su un apparato non dissimile da quello della villa c.d. 'catoniana'. L' evoluzione (o regressione) della grande proprietà fondiaria verso un sistema di piccole o medie aziende relativamente orientate anch' esse alla commercializzazione di parte della loro produzione, ma fondato su una responsabilità gestionale diffusa, non libera certo il dominus da quel coinvolgimento e da quelle responsabilità che lo stesso epistolario pliniano attesta, ma ne potenzia l' efficacia attraverso un impegno di un mumero di protagonisti a più livelli, nell' organizzazione della produzione agricola, maggiore di quanto il sistema schiavistico, nella sua forma 'pura', ne permettesse. 
Ma ir questo modo abbiamo già abbardonato gli aspetti più strettameste giuridici delle rimissiones e della locazione: quegli aspetti rispetto a cui questi primi paragrafi sono apparsi cosi strettamerte coincidere con le antiche impostazioni di De Neeve. Siamo ora passati a considerare piuttosto il furzionamento di tali istituzioni sotto il profilo socio-ecoromico. E qui, di nuovo, possono invece rilevarsi maggiori discrepanze fra $\mathrm{i}$ miei orientamerti di alcuri anni or sono e l' impostaziore dello stesso De Neeve, quale già affiorava nella sua opera maggiore sul Colonus e che più nettamente si è defirita ncl suc ultimo saggio. Un punto che firisce quasi con l' avere un valore strategico, in questo appare infatti la svalutazione della portata di alcunc testimonianze tardorepubblicane ai fini della preserza o mero di un ceto relativamente ampio di piccoli affittuari legati da rapporti di subalternità con i grandi proprietari. All' uopo De Neeve finiva infatti necessariamente col rilanciare l' importanza, ancora nel I sec. a.C., di altre figure arcaiche di dipendenza in modo che, a mio avviso, resta tuttcra assai discutibile.

Su questi aspetti si dovrà dunque tornare giacché, nella prospettiva qui rapidamente delireata, le mie conclusioni di un tempo, assumono nuovo valore. Sulla base della diversa e più trazidionale lettura dei testi discussi da De Neeve diventa infatti possibile trovare conferma alla presenza, in età repubblicana, di un sistema di piccoli affittuari legati a un ceto di grandi proprietari, confermandosi cosi, sin dall' inizio, la possibilità di quegli squilibri sociali che, invece, il compianto studioso olandese, soprattutto nella sua più recente ricerca del' 91 , tende fortemente a sottovalutare. 